\title{
The Functional State of Neutrophils Correlates With the Severity of Renal Dysfunction in Children With Hemolytic Uremic Syndrome
}

\author{
GABRIELA C. FERNANDEZ, SONIA A. GOMEZ, MARIA V. RAMOS, LETICIA V. BENTANCOR, \\ ROMINA J. FERNANDEZ-BRANDO, VERONICA I. LANDONI, LAURA LOPEZ, FLAVIA RAMIREZ, MARIO DIAZ, \\ MARTA ALDUNCIN, IRENE GRIMOLDI, RAMON EXENI, MARTIN A. ISTURIZ, AND MARINA S PALERMO
}

Division of Immunology [G.C.F., S.A.G., M.V.R., L.B., R.F.-B., V.L., M.A.I., M.S.P.], Institute of Hematological Investigations, National Academy of Medicine, C1425AUM Buenos Aires, Argentine; Department of Nephrology [M.A., I.G., R.E.], Pediatric Municipal Hospital of San Justo, B7118XAB, La Matanza, Buenos Aires, Argentine; Department of Nephrology [L.L., F.R., M.D.], Pediatric National Hospital

“Juan P. Garrahan”, C1249ABN, Buenos Aires, Argentine

\begin{abstract}
Hemolytic Uremic Syndrome (HUS) is the main cause of acute renal failure in children. The high percentage of patients who develop long-term sequelae constitutes an important medical concern. The identification of parameters that correlate with the degree of renal failure may be useful to plan the best treatment soon after hospitalization. Here, we investigated the functional state of neutrophils (PMN) from HUS patients on admission, before dialysis and/or transfusion, in relation to the severity of renal impairment reached during the acute period (AP). We found that all PMN activation parameters measured in severe cases of HUS (HUS AP3) were statistically lower comparing to children with mild cases of HUS (HUS AP1). As HUS PMN phenotype and dysfunction is compatible with that of cells undergoing cell death, we also studied spontaneous apoptosis. Not only were HUS PMN not apoptotic, but HUS AP3 PMN showed an increased survival. Almost all phenotypic and functional parameters measured on PMN correlated with severity. Our results revealed a marked deactivation of PMN in severe cases of HUS, and suggest that studying the functional state of PMN could be of prognostic value. (Pediatr Res 61: 123-128, 2007)
\end{abstract}

$\mathrm{H}^{\mathrm{e}}$ emolytic uremic syndrome (HUS) is a multi-systemic disorder characterized by the triad acute renal failure, microangiopathic hemolytic anemia and thrombocytopenia (1). HUS is the most common cause of acute renal failure and the second cause of chronic renal failure and renal transplantation in children (2). HUS is developed after a prodrome of hemorrhagic diarrhea caused by infection with Shiga toxin (Stx)-producing Escherichia coli (STEC) (3), and endothelial damage appears to be an important factor in the sequence of events leading to the microangiopathic process. No specific treatment for HUS is yet available, and although supportive therapies have reduced HUS mortality considerably, different degrees of renal insufficiency can be found in up to $40 \%$ of the patients (4-6). It has been reported that the main long-term prognostic factor is the severity of acute renal failure (4-7), as

Received May 18, 2006; accepted August 24, 2006.

Correspondence: Gabriela C. Fernández, Ph.D., Instituto de Investigaciones Hematológicas, Academia Nacional de Medicina, Pacheco de Melo 3081, C1425AUM, Buenos Aires, Argentina; e-mail: gfernandez@hematologia.anm.edu.ar

This work was supported by grants from Alberto J. Roemmers, Agencia Nacional de Promoción Científica y Tecnológica, and Consejo Nacional de Investigaciones Científicas y Tecnológicas (CONICET)

DOI: $10.1203 / 01 . p d r .0000250037 .47169 .55$ determined by the days of anuria, a criteria proposed by Gianantonio (8). Therefore, the identification of parameters that correlate with severity may be useful as possible prognostic factors.

HUS children usually show a high peripheral blood neutrophil (PMN) count at presentation, which has been correlated with a poor prognosis $(9-11)$. Moreover, increased levels of IL-8 have been detected in the serum of patients coursing the acute phase of HUS $(12,13)$. This cytokine is produced by activated PMN, promotes their adhesion and migration in vivo and shows anti-apoptotic properties (14-17). PMN are essential for host defense against microbial infections but can also be associated with the pathologic side effects of tissue destruction $(18,19)$. Potentially PMN-mediated harmful mechanisms include the production of toxic reactive oxygen species (ROS), and the proteolytic capacity of enzymes stored in granules. These storage organelles are not only a source of proteolytic or bactericidal proteins, but are also important reservoirs of membrane proteins that become incorporated into the surface membrane of PMN when these organelles fuse with the plasma membrane and exocytose their content. It is now established that, among other important receptors, the type III receptor form the Fc portion of the $\operatorname{IgG}(\mathrm{CD} 16)$ is stored in secretory vesicles, the adhesion molecule CD11b is mainly found in secretory vesicles and gelatinase granules, the degranulation marker CD66b is present in the membrane of specific granules, and the bactericidal enzyme myeloperoxidase (MPO) is stored in azurophil granules (20).

We have previously demonstrated that circulating PMN from HUS patients in the acute period (HUS AP) have a reduced expression of different membrane molecules and intracellular components, and also show impaired cytotoxic and cytokine-induced responses (21), indicating that PMN are partially deactivated, probably due to a postactivation exhaustion process $(17,18)$. However, the detailed analysis of the

\footnotetext{
Abbreviations: PMN, neutrophils; HUS, Hemolytic Uremic Syndrome; AP, acute period; HUS AP1, mild cases of HUS; HUS AP2, moderate cases of HUS; HUS AP3, severe cases of HUS; HC, healthy control; Stx, Shiga toxin; STEC, Stx-producing Escherichia coli; ROS, reactive oxygen species; MPO, myeloperoxidase; FITC, fluorescein isothiocyanate; DHR, dihydrorhodamine 123; PMA, phorbol myristate acetate
} 
relationship between the functional state of PMN and the severity of the renal dysfunction of the patients has not been previously investigated. In this study, we analyzed in HUS patients on admission whether the level of deactivation of PMN correlates with the degree of renal impairment. In particular, we determined the expression of activation markers situated in different types of intracellular granules, as well as ROS generation. We also investigated if deactivation of peripheral PMN from HUS AP patients was a consequence of an increased number of cells undergoing apoptosis.

\section{METHODS}

Patients and samples. The study was approved by the respective Hospital Ethical Committees. Patients $(n=50)$ were enrolled in this study between January, 2000 and December, 2005 after informed consent from their parents had been obtained. The criteria for diagnosis were a microangiopathic hemolytic anemia with schizocytes, thrombocytopenia (platelet count $<150 \times$ $10^{9} / \mathrm{L}$ ) and acute renal failure (serum creatinine level higher than the normal values considering the age of the patient). All patients develop HUS after gastroenteritis consisting of bloody diarrhea. $60 \%$ of the children were positive for STEC, diagnosed by stool culture and/or presence of Stx-antibody in serum. Twenty-four were girls and 26 were boys. Clinical and biochemical data of patients are given in Table 1 . Blood samples $(2 \mathrm{~mL})$ were obtained for biochemical and immunologic studies by venopuncture into EDTA plastic tubes, before dialysis and/or transfusion, at different days from the time when diarrhea have started (Table 1). 31 patients received peritoneal dialysis and one received hemodialysis within $24 \mathrm{~h}$ since admission, but after the blood sample was collected. The amount of blood obtained from each patient was not enough to measure all the parameters evaluated in this study, and the number of children analyzed is indicated in each figure. Blood samples from age- and sex-matched healthy children (HC) admitted for routine surgical procedures were collected and processed identically.

Immunophenotypic studies. Measurement of the surface expression of CD16, CD11b and CD66 was performed by direct immunofluorescence flow cytometry using the following conjugated mouse anti-human MAb (MAb): CD16-PE (R-phycoerythrin, BD Biosciences, San Jose, CA), CD11b-PE (Immunotech) and CD66-FITC (FITC, Immunotech). Whole blood 100- $\mu \mathrm{L}$ samples were incubated with the specific MAb for $30 \mathrm{~min}$ at room temperature. Then, erythrocytes were lysed using FACS Lysing solution (BD Biosciences), and cells were re-suspended in $0.2 \mu \mathrm{L}$ of ISOFLOW (International Link, Buenos Aires, Argentina). The total content of MPO was determined following the instructions of Fix \& Perm Cell Permeabilization Kit. Briefly, cells were fixed in suspension, washed and then permeabilized in the presence of specific antibody. Then, cells were washed and suspended in $0.2 \mu \mathrm{L}$ of ISOFLOW. In all cases control of isotype-matched antibody was assayed in parallel and the fluorescence was measured with a Becton Dickinson FACS- can. The analysis was made on 10,000 events on each sample using the Cell Quest program. PMN were identified and gated according to their forward and light scattering (FSC/SSC) dot-plot profiles.

Pmn isolation. PMN were isolated by Ficoll-Hypaque gradient centrifugation (Ficoll Pharmacia, Uppsala; Hypaque, Wintthrop Products, Buenos Aires, Argentina) and dextran sedimentation, as previously described (22).

Reactive oxygen species (ROS) generation. Purified $0.25 \times 10^{6}$ of PMN were incubated with dihydrorhodamine 123 (DHR, Sigma Chemical Co.) $(1 \mathrm{mM})$ at $37^{\circ} \mathrm{C}$ in $5 \% \mathrm{CO}_{2}$. After $15 \mathrm{~min}$, phorbol myristate acetate (PMA, $20 \mathrm{ng} / \mathrm{mL}$ ) was added and incubated for 15 additional min. Upon ROS generation a brightly fluorescent FL-1 product is produced, which was detected by flow cytometry.

Spontaneous apoptosis. Isolated PMN $\left(0.5 \times 10^{6}\right.$ cells $)$ were incubated for $18 \mathrm{~h}$ at $37^{\circ} \mathrm{C}$ in $5 \% \mathrm{CO}_{2}$. FITC-labeled annexin $\mathrm{V}(1 \mu \mathrm{g} / \mathrm{mL})$ was added for $1 \mathrm{~h}$ at $4^{\circ} \mathrm{C}$ to cells incubated in HEPES buffer (HEPES $10 \mathrm{mM}, \mathrm{pH} 7.4 ; \mathrm{NaCl}$ $150 \mathrm{mM} ; \mathrm{KCl} 5 \mathrm{mM} ; \mathrm{MgCl}_{2} 1 \mathrm{mM} ; \mathrm{CaCl}_{2} 1.8 \mathrm{mM}$ ). Cells were washed, re-suspended in HEPES buffer, and incubated for 10 min with propidium iodide (PI, $15 \mu \mathrm{g} / \mathrm{mL}$ ). The percentage of simple and/or double positive cells was determined by flow cytometry. Spontaneous apoptosis was also measured on isolated PMN from normal adult donors in the presence of $15 \%$ of HUS or HC plasma.

Il-8 determination. IL-8 determination was performed on $100 \mu \mathrm{L}$ of plasma by sandwich ELISA. Briefly, an ELISA 96-well plate (Greiner-Bio One, Frickenhausen, Germany) was covered overnight with $5 \mu \mathrm{g} / \mathrm{mL}$ of anti-IL-8 capture antibody (BD Pharmingen). Samples were added and incubated at room temperature for $2 \mathrm{~h}$. The biotinylated anti-cytokine detection antibody $(2 \mu \mathrm{g} / \mathrm{mL}$, BD Pharmingen) was added followed by a 1:10,000 dilution of streptavidin-horseradish peroxidase (Caltag Laboratories) and a solution of $0.4 \mathrm{mg} / \mathrm{mL}$ o-phenylene-diamine (OPD, Sigma Chemical Co.) in $0.1 \mathrm{M}$ citric acid, $0.05 \mathrm{M} \mathrm{Na}_{2} \mathrm{HPO}_{4}, 3 \% \mathrm{H}_{2} \mathrm{O}_{2}$. The reaction was stopped with $\mathrm{H}_{2} \mathrm{SO}_{4} 2 \mathrm{M}$ and the resultant OD read at $492 \mathrm{~nm}$. The amount of IL- $8(\mathrm{pg} / \mathrm{mL})$ was extrapolated using a standard curve constructed over the range of $25-1000 \mathrm{pg} / \mathrm{mL}$ of human recombinant IL-8 (BD Pharmingen).

Statistics. Comparisons between groups were performed using the nonparametric unpaired Mann-Whitney test. For correlation analysis the nonparametric, two-tailed Spearman's rank correlation test was used. $p<0.05$ was considered significant.

\section{RESULTS}

Expression of molecules present in different types of granules of PMN. We studied different markers of activation on PMN from HUS patients on admission, before dialysis and/or transfusion. According to Gianantonio's criteria (8), patients were classified according to the severity of renal dysfunction as mild cases (HUS AP1 no anuria), moderate cases (HUS AP2 $<7 \mathrm{~d}$ of anuria), or severe cases (HUS AP3

Table 1. Clinical and biochemical data of HUS patients

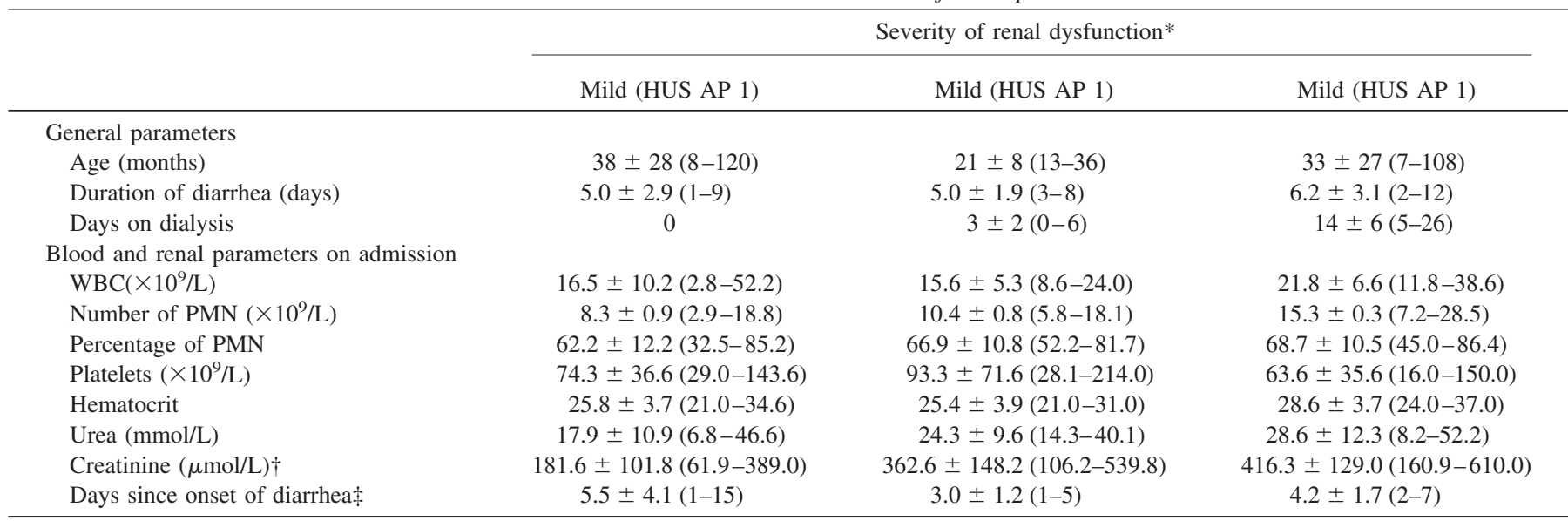

* According to Gianantonio's criteria (8), patients were classified considering their severity of renal dysfunction as mild cases (HUS AP1 no anuria), moderate cases (HUS AP2 $<7$ days of anuria), or severe cases (HUS AP3 $>7$ days of anuria); $†$ Creatinine was determine using the kinetic Jaffe alkaline picrate colorimetric method; $\ddagger$ Days after onset of diarrhea that blood was obtained for the analysis reported.

WBC, white blood cell count. 
$>7 \mathrm{~d}$ of anuria). Figure $1 A$ shows that the surface expression of CD16 was equally decreased in all HUS groups compared with healthy children $(\mathrm{HC})$ without differences within the HUS population. On the other hand, although all HUS groups showed a decreased CD11b expression compared with $\mathrm{HC}$ children, we found a gradual CD11b decreased expression as the severity of HUS increased, resulting in statistically significant differences between HUS AP1 and HUS AP3 groups (Fig. 1B).

The expression of CD66b in HUS AP1 children was similar to HC, and HUS AP2 and 3 showed a decreased expression of this molecule compared with HUS AP1, although the differences between HUS AP3 patients and HC children did not reach statistical significance (Fig. 2A). Similar results were obtained for the total content of MPO, which was found decreased in HUS AP2 and 3 groups compared with HUS AP1 (Fig. 2B). Although MPO total content of HUS AP2 and 3 was also decreased compared with $\mathrm{HC}$, statistical differences between HUS AP2 and HC were marginal $(p=0.06)$.

Reactive oxygen species (ROS) generation by PMN from HUS patients. ROS production is another indicator of the functional state of PMN. We found no differences on basal ROS generation between groups (data not shown). However, PMA-triggered ROS production was found decreased only in HUS AP3 PMN, and this impairment was statistically significant compared with all groups (Fig. 3).

Spontaneous apoptosis of PMN from HUS patients. We measured spontaneous apoptosis on isolated PMN using FITC-conjugated annexin V and propidium iodide (PI) (Fig. 4). Different subpopulations can be identified using this technique: double negative cells correspond to live cells, double positive cells to late apoptotic cells, and positive cells for annexin V only, to early apoptotic state. As shown in Fig. 4B, we found that the percentage of early apoptotic PMN was decreased in all HUS groups compared with HC. Moreover,
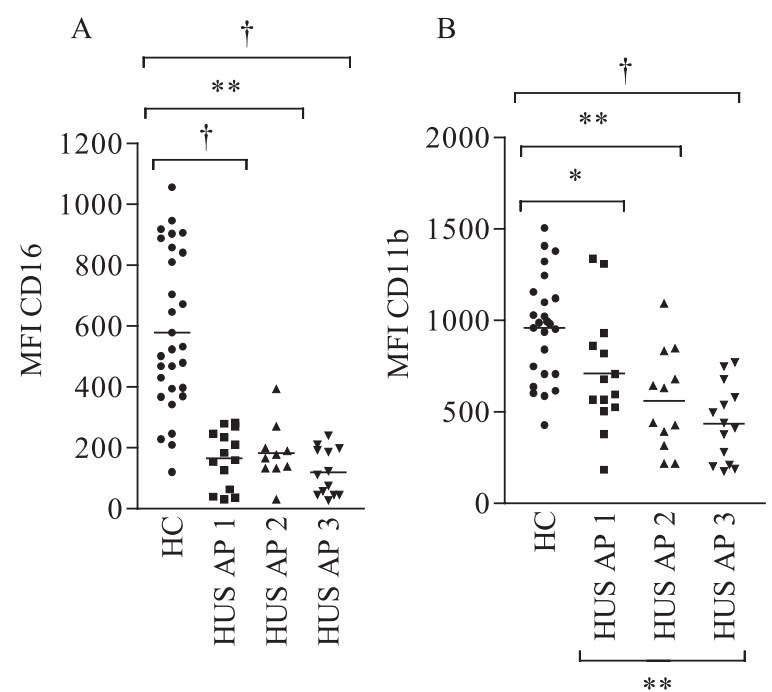

Figure 1. $(A)$ The mean fluorescence intensity (MFI) of CD16 was measured in HC $(n=29)$, HUS AP1 $(n=14)$, HUS AP2 $(n=10$ and HUS AP3 $(n=$ 13). (B) The surface expression of CD11b was determined in HC $(n=25)$, HUS AP1 $(n=16)$, HUS AP2 $(n=12)$ and HUS AP3 $(n=14)$. Each point represents individual values and the horizontal line the mean value. ${ }^{*} p<$ $0.05, * * p<0.01$ and $\dagger p<0.001$.
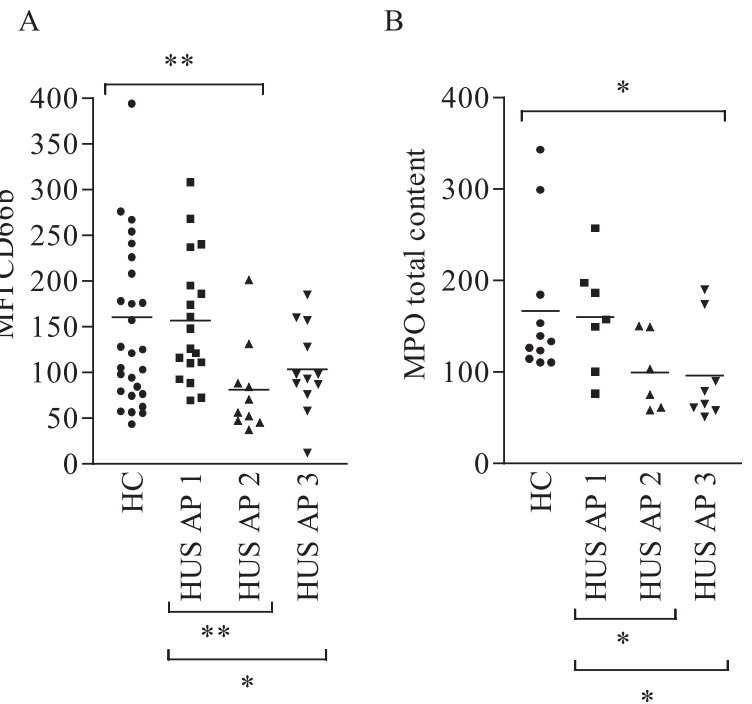

Figure 2. (A) CD66b surface expression was determined in HC $(n=27)$, HUS AP1 $(n=18)$, HUS AP2 $(n=10)$ and HUS AP3 $(n=12)$. $(B)$ The total content of MPO was determined in HC $(n=11)$, HUS AP1 $(n=7)$, HUS AP2 $(n=6)$ and HUS AP3 $(n=8)$. Each point represents individual values and the horizontal line the mean value. ${ }^{*} p<0.05$ and $* * p<0.01$.

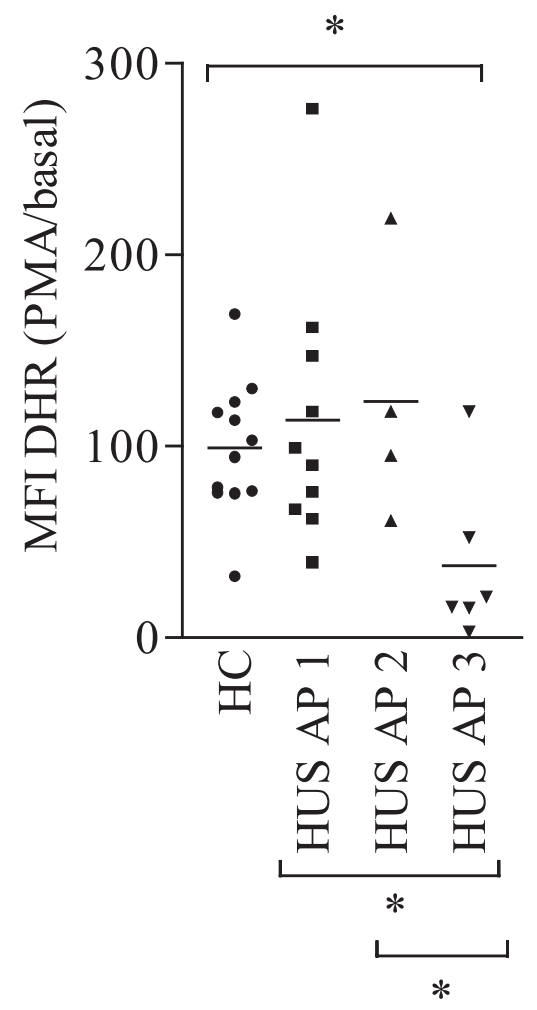

Figure 3. ROS production was measured in HC $(n=12)$, HUS AP1 $(n=$ $10)$, HUS AP2 $(n=4)$ and HUS AP3 $(n=6)$. Results were expressed as the MFI of DHR in the presence of PMA with respect to the MFI of DHR in basal conditions. Each point represents individual values and the horizontal line the mean value. ${ }^{*} p<0.05$.

PMN from HUS AP3 showed lower levels of apoptosis than HUS AP1. Regarding late apoptosis (Fig. 4C), only children with severe renal impairment (HUS AP3) evidenced a statistically significant decreased percentage in late apoptotis compared with HC and HUS AP1. Conversely, the percentage of live cells (Fig. 4D) was higher in HUS AP3 compared with HC and HUS AP1. 

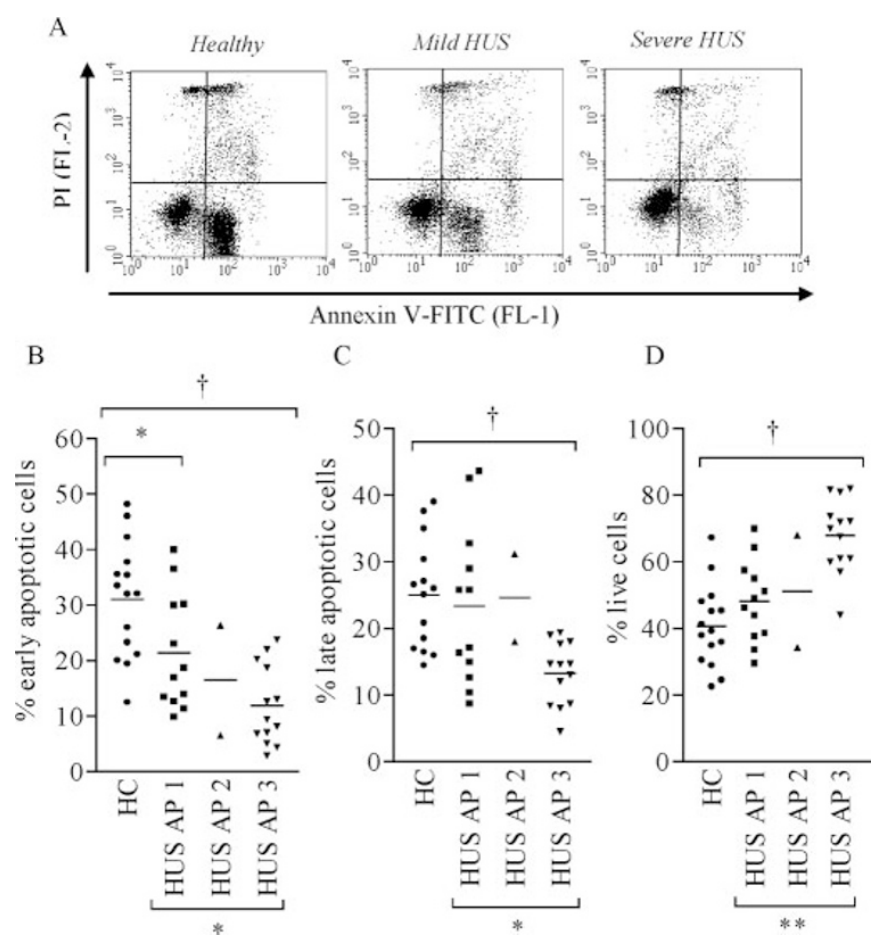

Figure 4. Spontaneous apoptosis was measured in HC $(n=15)$, HUS AP1 $(n=12)$, HUS AP2 $(n=2)$ and HUS AP3 $(n=13)$. (A) Representative dot plots of one HC, one HUS AP1 and one HUS AP3 child showing the different apoptotic subpopulations. (B) Early apoptotic PMN. (C) Late apoptotic PMN. (D) Live cells. Each point represents individual values and the horizontal line the mean value. ${ }^{*} p<0.05 ; * p<0.01 ; \dagger p<0.001$.

To study the contribution of soluble factors in the inhibition of apoptosis observed in HUS patients, isolated PMN from normal adult donors $(n=3)$ were incubated for $18 \mathrm{~h}$ in the presence of plasma from HUS AP1 patients $(n=4)$, HUS AP3 patients $(n=8)$ or $\mathrm{HC}$ children $(n=12)$. We found that the percentage of apoptosis of adult PMN was similar when incubated with plasma from HUS or HC children (early apoptosis: $\mathrm{HC}=19.8 \pm 4.3 \%$, HUS AP1 $=19.9 \pm 5.2 \%$, HUS AP3 $=18.2 \pm 4.5 \%$; late apoptosis: $\mathrm{HC}=7.5 \pm 1.3 \%$, HUS AP1 $=15.5 \pm 5.9 \%$, HUS AP3 $=7.9 \pm 1.6 \%$; live cells: $\mathrm{HC}=70.6 \pm 3.2 \%$, HUS AP1 $=66.0 \pm 6.1 \%$, HUS $\mathrm{AP} 3=71.9 \pm 3.7 \%)$.

Plasmatic IL-8 levels in HUS patients. When we evaluated plasmatic IL-8 we found that, in agreement with other reports $(12,13)$, the entire group of HUS AP children showed higher levels of plasmatic IL-8 compared with HC (pg/mL of IL-8: $\mathrm{HC}=338.5 \pm 53.9$, HUS A $p=966.5 \pm 126.9, p<0.01)$. However, when HUS children were divided according to their severity, we found that only HUS AP1 showed a statistically significant increase in IL-8 levels compared with HC and HUS AP3 (Fig. 5).

Correlations. Biochemical and functional parameters measured on PMN were correlated with severity (Table 2). The white blood cell count (WBC), the absolute number and percentage of PMN and the percentage of live PMN showed positive correlations with severity, whereas the expression of different activation markers (CD11b, CD66b and MPO), ROS production capacity, percentage of apoptosis and plasmatic IL-8 levels showed negative correlations with severity.

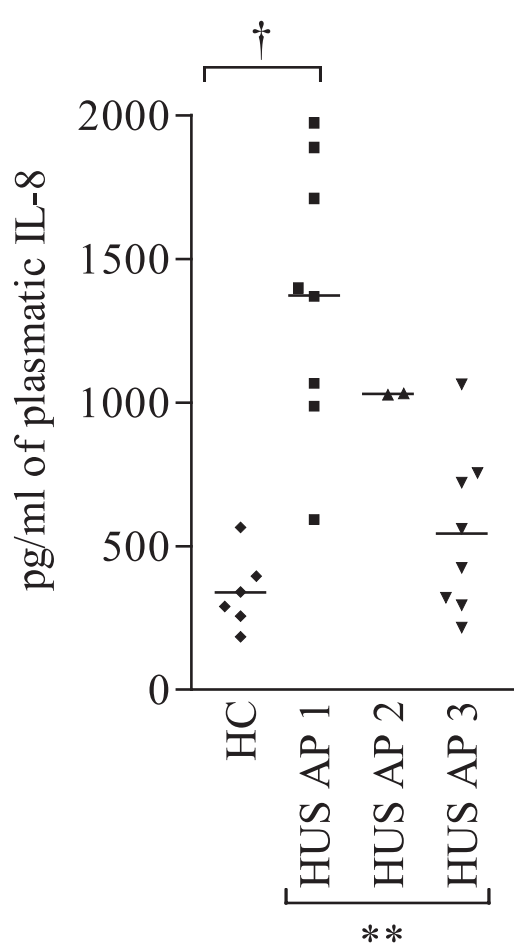

Figure 5. IL-8 plasmatic levels $(\mathrm{pg} / \mathrm{mL})$ were determined in $\mathrm{HC}(n=6)$, HUS AP1 $(n=8)$, HUS AP2 $(n=2)$ and HUS AP3 $(n=8)$. Each point represents individual values and the horizontal line the mean value. $* * p<$ $0.01 ; \dagger p<0.001$.

Table 2. Correlation data of the different parameters measured in $P M N$ on admission and the severity of renal dysfunction during the acute period in HUS patients

\begin{tabular}{lcc}
\hline & Pearson's $r$ & Significance $(p$-value $)$ \\
\hline WBCn $n=50$ & $r=0.39$ & $p=0.004$ \\
Number of PMN $n=50$ & $r=0.62$ & $p=0.001$ \\
Percentage of PMN $n=50$ & $r=0.28$ & $p=0.04$ \\
Expression of & \multicolumn{2}{c}{ No correlation } \\
CD16n $n=40$ & $r=-0.37$ & $p=0.01$ \\
CD11b $n=40$ & $r=-0.35$ & $p=0.01$ \\
CD66b $n=40$ & $r=-0.51$ & $p=0.01$ \\
MPO $n=21$ & $r=-0.51$ & $p=0.01$ \\
ROS generation $n=21$ & & \\
Percentage of cells & $r=-0.44$ & $p=0.02$ \\
Early apoptosis $n=27$ & $r=-0.43$ & $p=0.02$ \\
Late apoptosis $n=27$ & $r=0.63$ & $p<0.001$ \\
Live cells $n=27$ & $r=-0.74$ & $p<0.001$ \\
Plasmatic IL-8 levels $n=18$ & &
\end{tabular}

Considering the anti-apoptotic properties of IL-8, we determined the correlation between the rate of apoptosis and IL- 8 levels in HUS AP patients. There was no correlation between plasmatic IL-8 levels and spontaneous apoptosis of PMN. However, a positive correlation was found between ROS generation and the percentage of late apoptotic cells in HUS $(r=0.66, p=0.01)$.

\section{DISCUSSION}

Through several years of investigation it has been established that HUS has a very important inflammatory component that contributes to the pathogenesis of the disease. Thus, reports of PMN leukocytosis in HUS are not surprising, since 
PMN are prominent components of the acute inflammatory response. A high peripheral PMN count at presentation has been largely associated with a poor prognosis (9-11). Our results also support these observations as patients with more severe renal impairment showed the highest PMN count. Paradoxically, an inverse relation was observed between severity and the functional state of PMN: whereas mild cases of HUS (HUS AP1) showed some decreased but mostly unaffected parameters of PMN activation, PMN from severe cases of HUS (HUS AP3) evidenced significant decreases in these parameters. As the phenotypic and functional characteristics observed in HUS PMN have been associated with apoptosis $(23,24)$, we investigated whether HUS PMN could be undergoing cell death. Not only were HUS PMN not apoptotic, but more deactivated PMN showed an increased survival. The lower levels in spontaneous apoptosis found in severe cases of HUS could contribute, at least in part, to the neutrophilia usually observed in these patients. PMN are a very important source of cytokines in vivo, including IL-8, a cytokine that activates PMN and inhibits their apoptosis $(17,25,26)$. However, IL-8 seems not to be involved in preventing apoptosis of HUS PMN, since there was no correlation between the plasmatic level of IL-8 and the percentage of apoptosis. Moreover, apoptosis of PMN from normal adult donors was similar in the presence of HUS AP1 plasma, which presented the highest levels of IL-8, HUS AP3 plasma or normal plasma. Interestingly, only mild HUS patients showed increased levels of plasmatic IL-8, compatible with the absence of deactivation observed in PMN from HUS AP1 compared with HUS AP3. Whether the lower levels of IL-8 observed in HUS AP3 compared with HUS AP1 result from an impaired production by deactivated PMN needs further investigation. On the other hand, the positive correlation found between ROS generation and the percentage of apoptotic cells suggests that the impaired capacity of these cells to produce ROS could increase PMN survival. Accordingly, several studies have demonstrated that the presence of ROS scavengers inhibits PMN apoptosis $(27,28)$.

Most of the activation markers measured in this study reside in the plasma membrane and/or in intracellular granules. The activation of PMN results in the mobilization of these granules, leading to a rapid up-regulation of these molecules followed by their down-regulation in the plasma membrane (29). This process of exocytosis is mediated by different mechanisms $(30,31)$, and can explain the decreased expression of PMN membrane antigens found in HUS patients. According to this process, our previous data reporting a decreased intracellular content of enzymes and antigens, supports the concept that PMN from HUS patients have previously been activated and degranulated $(21,32)$. Moreover, PMN-derived proteases in serum have been found in HUS patients (33). Each activation marker measured on PMN from HUS patients showed a different pattern of expression within HUS severity groups. Although CD16 expression was equally decreased in all groups, the majority of the markers (CD11b, CD66b and MPO) showed a gradual decrease as the severity progressed from HUS AP1 to HUS AP3. It is well established that there are major differences between the granule subsets regarding the extent to which these are mobilized both in vitro and in vivo $(29,34)$. Secretory vesicles (CD16, CD11b) are mobilized more readily than specific (CD66b) and azurophil (MPO) granules $(20,35)$. This hierarchy in granule mobilization and the extent of exocytosis was found to be quantitatively related to the stimulus strength (35). Our results showed that in HUS AP1 the expression and content of the markers found in specific and azurophil granules was not different from that of $\mathrm{HC}$, although lower levels of these molecules were observed in HUS AP2 and 3. We can hypothesize that the PMN activating stimulus acting in mild cases is sufficient to cause degranulation of the more rapidly mobilized intracellular structures, the secretory vesicles, but not enough to affect specific and azurophil granules. Accordingly, PMN from HUS AP3 have experienced a more generalized process of degranulation as a consequence of a previous stronger stimulus.

Although there is overlapping between values in children of the different severity groups, many variables may be contributing to the functional state of PMN, and all these variables may vary between individuals. HUS patients probably differ in the initial bacterial inoculum, the Stx producing capacity of the bacteria ingested, the specific characteristic and degree of activation of the thrombotic and inflammatory responses, and the individual variability. As the overlapping can limit the prognostic value of the evaluation of the functional state of PMN, the entire pattern of PMN activation instead of one single parameter has to be considered.

HUS is a systemic multi-factorial complication of STEC infections, probably arising from lesions caused by circulating Stx. While the vascular injury leading to HUS is likely to be in progress at the time of hospitalization, much of the thrombotic damage has yet occurred (36-38). Indeed, it is well known that children with HUS on admission showed thrombocytopenia as well as degranulated platelets, secondary to a strong thrombotic stimulus $(39,40)$. Parallel to these findings, our hypothesis is that circulating PMN on admission are low responders as a consequence of a previous activation process, which triggered the respiratory burst and the release of proteases associated to degranulation, leading to deactivation or exhaustion of PMN. These two mechanisms may actively contribute to the endothelial injury initiated by the direct insult caused by Stx. Endothelial cell damage results in the upregulation of adhesion molecules and the release of inflammatory and prothrombotic mediators that will in turn activate platelets and PMN. Systemically activated PMN could adhere to the endothelium, but will fail to transmigrate in the absence of an inflammatory focus and will be retain in circulation in a deactivated state. In this regard, PMN adhesion and detachment to endothelial cells has been reported (41). In line with this hypothesis, we propose that a strong initial activating stimulus will induce a strong activation of PMN that in turn will derive in a more severe clinical course. Consequently, we found a more deactivated state of PMN on admission in HUS AP3 children. This process of PMN impairment may be implicated in limiting further damaging mechanisms. In this sense, infection, injury and/or isquemia results in the release of pro-inflammatory mediators, and this response is then downregulated by the release of anti-inflammatory mediators, re- 
sulting in the restoration of homeostasis. In fact, HUS patients consistently showed increased levels of anti-inflammatory cytokines, which have been associated with a more severe renal dysfunction of patients (42-44).

In conclusion, in this study we determined that all activation parameters measured on PMN from HUS AP3 were significantly decreased compared with HUS AP1 and HC. The close correlation found between PMN deactivation on admission and the severity of renal dysfunction achieved during the acute period suggests that the analysis of the functional state of PMN in the acute period can be useful for assessing prognosis.

Acknowledgments. The authors thank Nora Galassi, Marta Felippo and Norma Riera, for their excellent technical assistance, and Fabiana Alberto and Emilse Bermejo for normal adult donor samples.

\section{REFERENCES}

1. Remuzzi G, Ruggenenti P 1995 The hemolytic uremic syndrome. Kidney Int 48:2-19

2. Kaplan BS, Meyers KE, Schulman SL 1998 The pathogenesis and treatment of hemolytic uremic syndrome. J Am Soc Nephrol 9:1126-1133

3. Paton JC, Paton AW 1998 Pathogenesis and diagnosis of Shiga toxin-producing Escherichia coli infections. Clin Microbiol Rev 11:450-479

4. Spizzirri FD, Rahman RC, Bibiloni N, Ruscasso JD, Amoreo OR 1997 Childhood hemolytic uremic syndrome in Argentina: long-term follow-up and prognostic features. Pediatr Nephrol 11:156-160

5. de Jong M, Monnens L 1988 Haemolytic-uraemic syndrome: a 10-year follow-up study of 73 patients. Nephrol Dial Transplant 3:379-382

6. Siegler RL, Milligan MK, Burningham TH, Christofferson RD, Chang SY, Jorde LB 1991 Long-term outcome and prognostic indicators in the hemolytic-uremic syndrome. J Pediatr 118:195-200

7. Huseman D, Gellermann J, Vollmer I, Ohde I, Devaux S, Ehrich JH, Filler G 1999 Long-term prognosis of hemolytic uremic syndrome and effective renal plasma flow. Pediatr Nephrol 13:672-677

8. Gianantonio CA, Vitacco M, Mendilaharzu F, Gallo GE, Sojo ET 1973 The hemolytic-uremic syndrome. Nephron 11:174-192

9. Fitzpatrick MM, Shah V, Filler G, Dillon MJ, Barratt TM 1992 Neutrophil activation in the haemolytic uraemic syndrome: free and complexed elastase in plasma. Pediatr Nephrol 6:50-53

10. Milford D, Taylor CM, Rafaat F, Halloran E, Dawes J 1989 Neutrophil elastases and haemolytic uraemic syndrome. Lancet 2:1153

11. Robson WL, Fick GH, Wilson PC 1988 Prognostic factors in typical postdiarrhea hemolytic-uremic syndrome. Child Nephrol Urol 9:203-207

12. Fitzpatrick MM, Shah V, Trompeter RS, Dillon MJ, Barratt TM 1992 Interleukin-8 and polymorphoneutrophil leucocyte activation in hemolytic uremic syndrome of childhood. Kidney Int 42:951-956

13. van Setten PA, van Hinsbergh VW, van den Heuvel LP, Preyers F, Dijkman HB, Assmann KJ, van der Velden TJ, Monnens LA 1998 Monocyte chemoattractant protein- 1 and interleukin- 8 levels in urine and serum of patents with hemolytic uremic syndrome. Pediatr Res 43:759-767

14. Scapini P, Lapinet-Vera JA, Gasperini S, Calzetti F, Bazzoni F, Cassatella MA 2000 The neutrophil as a cellular source of chemokines. Immunol Rev 177:195-203

15. Detmers PA, Lo SK, Olsen-Egbert E, Walz A, Baggiolini M, Cohn ZA 1990 Neutrophil-activating protein 1/interleukin 8 stimulates the binding activity of the leukocyte adhesion receptor CD11b/CD18 on human neutrophils. J Exp Med 171:1155-1162

16. Huber AR, Kunkel SL, Todd RF 3rd, Weiss SJ 1991 Regulation of transendothelial neutrophil migration by endogenous interleukin-8. Science 254:99-102

17. Kettritz R, Gaido ML, Haller H, Luft FC, Jennette CJ, Falk RJ 1998 Interleukin-8 delays spontaneous and tumor necrosis factor-alpha-mediated apoptosis of human neutrophils. Kidney Int 53:84-91

18. Weiss SJ 1989 Tissue destruction by neutrophils. N Engl J Med 320:365-376
19. Lentsch AB, Ward PA 2000 Regulation of inflammatory vascular damage. J Pathol 190:343-348

20. Borregaard N, Cowland JB 1997 Granules of the human neutrophilic polymorphonuclear leukocyte. Blood 89:3503-3521

21. Fernandez GC, Gomez SA, Rubel CJ, Bentancor LV, Barrionuevo P, Alduncin M, Grimoldi I, Exeni R, Isturiz MA, Palermo MS 2005 Impaired neutrophils in children with the typical form of hemolytic uremic syndrome. Pediatr Nephrol 20:1306-1314

22. Boyum A 1968 Separation of leukocytes from blood and bone marrow. Introduction. Scand J Clin Lab Invest Suppl 97:7

23. Dransfield I, Buckle AM, Savill JS, McDowall A, Haslett C, Hogg N 1994 Neutrophil apoptosis is associated with a reduction in CD16 (Fc gamma RIII) expression. J Immunol 153:1254-1263

24. Whyte MK, Meagher LC, MacDermot J, Haslett C 1993 Impairment of function in aging neutrophils is associated with apoptosis. J Immunol 150:5124-5134

25. Xing Z, Jordana M, Kirpalani H, Driscoll KE, Schall TJ, Gauldie J 1994 Cytokine expression by neutrophils and macrophages in vivo: endotoxin induces tumor necrosis factor-alpha, macrophage inflammatory protein-2, interleukin-1 beta, and interleukin-6 but not RANTES or transforming growth factor-beta 1 mRNA expression in acute lung inflammation. Am J Respir Cell Mol Biol 10:148-153

26. Malazdrewich C, Ames TR, Abrahamsen MS, Maheswaran SK 2001 Pulmonary expression of tumor necrosis factor alpha, interleukin-1 beta, and interleukin- 8 in the acute phase of bovine pneumonic pasteurellosis. Vet Pathol 38:297-310

27. Rollet-Labelle E, Grange MJ, Elbim C, Marquetty C, Gougerot-Pocidalo MA, Pasquier C 1998 Hydroxyl radical as a potential intracellular mediator of polymorphonuclear neutrophil apoptosis. Free Radic Biol Med 24:563-572

28. Lundqvist-Gustafsson H, Bengtsson T 1999 Activation of the granule pool of the NADPH oxidase accelerates apoptosis in human neutrophils. J Leukoc Biol 65:196204

29. Sengelov H, Follin P, Kjeldsen L, Lollike K, Dahlgren C, Borregaard N 1995 Mobilization of granules and secretory vesicles during in vivo exudation of human neutrophils. J Immunol 154:4157-4165

30. Beigier-Bompadre M, Barrionuevo P, Alves-Rosa F, Rubel CJ, Palermo MS, Isturiz MA 2003 The formyl peptide N-formyl-methionyl-leucyl-phenylalanine downregulates the expression of FcgammaRs in interferon-gamma-activated monocytes/ macrophages in vitro and in vivo. Scand J Immunol 57:221-228

31. Bazil V, Strominger JL 1991 Shedding as a mechanism of down-modulation of CD14 on stimulated human monocytes. J Immunol 147:1567-1574

32. Fernandez GC, Rubel C, Barrionuevo P, Lopez L, Ramirez F, Diaz M, Isturiz MA, Palermo MS 2002 Phenotype markers and function of neutrophils in children with hemolytic uremic syndrome. Pediatr Nephrol 17:337-344

33. Kaplan BS, Mills M 1988 Elevated serum elastase and alpha-1-antitrypsin levels in hemolytic uremic syndrome. Clin Nephrol 30:193-196

34. Wright DG 1988 Human neutrophil degranulation. Methods Enzymol 162:538-551

35. Lew PD, Monod A, Waldvogel FA, Dewald B, Baggiolini M, Pozzan T 1986 Quantitative analysis of the cytosolic free calcium dependency of exocytosis from three subcellular compartments in intact human neutrophils. J Cell Biol 102:21972204

36. Mori Y, Wada H, Okugawa Y, Tamaki S, Nakasaki T, Watanabe R, Gabazza EC, Nishikawa M, Minami N, Shiku H 2001 Increased plasma thrombomodulin as a vascular endothelial cell marker in patients with thrombotic thrombocytopenic purpura and hemolytic uremic syndrome. Clin Appl Thromb Hemost 7:5-9

37. Nevard CH, Jurd KM, Lane DA, Philippou H, Haycock GB, Hunt BJ 1997 Activation of coagulation and fibrinolysis in childhood diarrhoea- associated haemolytic uraemic syndrome. Thromb Haemost 78:1450-1455

38. Tarr PI, Gordon CA, Chandler WL 2005 Shiga-toxin-producing Escherichia coli and haemolytic uraemic syndrome. Lancet 365:1073-1086

39. Fong JS, Kaplan BS 1982 Impairment of platelet aggregation in hemolytic uremic syndrome: evidence for platelet "exhaustion". Blood 60:564-570

40. Walters MD, Levin M, Smith C, Nokes TJ, Hardisty RM, Dillon MJ, Barratt TM 1988 Intravascular platelet activation in the hemolytic uremic syndrome. Kidney Int 33:107-115

41. Luu NT, Rainger GE, Nash GB 1999 Kinetics of the different steps during neutrophil migration through cultured endothelial monolayers treated with tumour necrosis factor-alpha. J Vasc Res 36:477-485

42. Litalien C, Proulx F, Mariscalco MM, Robitaille P, Turgeon JP, Orrbine E, Rowe PC, McLaine PN, Seidman E 1999 Circulating inflammatory cytokine levels in hemolytic uremic syndrome. Pediatr Nephrol 13:840-845

43. van de Kar NC, Sauerwein RW, Demacker PN, Grau GE, van Hinsbergh VW, Monnens LA 1995 Plasma cytokine levels in hemolytic uremic syndrome. Nephron 71:309-313

44. Proulx F, Turgeon JP, Litalien C, Mariscalco MM, Robitaille P, Seidman E 1998 Inflammatory mediators in Escherichia coli O157:H7 hemorrhagic colitis and hemolytic-uremic syndrome. Pediatr Infect Dis J 17:899-904 